\title{
Health-related Quality of Life in Adult Patients with Morbid Obesity Coming for Bariatric Surgery
}

\author{
Chi-Yang Chang $\cdot$ Chih-Kun Hung • Yu-Yin Chang • \\ Chi-Ming Tai $\cdot$ Jaw-Town Lin $\cdot$ Jung-Der Wang
}

Received: 16 March 2008 /Accepted: 19 March 2008 /Published online: 8 May 2008

(C) The Author(s) 2008

\begin{abstract}
Background Obesity has become a major health issue not only in the West but also in Asia. Morbid obesity can lead to much comorbidity and can markedly interfere with quality of life. The aim of this study was to compare the health-related quality of life (HRQL) between patients with morbid obesity coming for bariatric surgery and the healthy population in Taiwan.

Methods Patients were between age 18 and 65 years. Patients had a BMI between 32 and $40 \mathrm{~kg} / \mathrm{m}^{2}$ with obesity-related comorbidities or a BMI $>40 \mathrm{~kg} / \mathrm{m}^{2}$. Patients were enrolled for bariatric surgery by a modified recommendation of the Asia-Pacific consensus. Physical and psychiatric evaluations were accomplished simultaneously. The World Health Organization Quality of Life (WHOQOLBREF), Taiwan version, was administered 1 month before the operation. The quality of life of the obese patients was
\end{abstract}

C.-Y. Chang $\cdot$ C.-M. Tai $\cdot$ J.-T. Lin

Department of Internal Medicine, E-Da Hospital,

I-Shou University, Kaohsiung, Taiwan, ROC

C.-K. Hung

Department of Surgery, E-Da Hospital, I-Shou University,

Kaohsiung, Taiwan, ROC

C.-Y. Chang $\cdot$ J.-T. Lin $\cdot$ J.-D. Wang

Department of Internal Medicine,

National Taiwan University Hospital, Taipei, Taiwan, ROC

C.-Y. Chang $\cdot$ Y.-Y. Chang $\cdot$ J.-D. Wang $(\bowtie)$

Institute of Occupation Medicine and Industrial Hygiene, College of Public Health, National Taiwan University,

Rm. 719, No. 17, Shiujou Rd., Taipei, Taiwan, ROC

e-mail: jdwang@ntu.edu.tw

J.-D. Wang

Department of Environmental and Occupational Medicine,

National Taiwan University Hospital, Taipei, Taiwan, ROC compared with age-, sex-, education-, marriage-, and municipality-matched healthy control patients taken from a national survey in Taiwan. Multiple regression analyses were conducted to study risk factors for impairment of HRQL. Results A total of 114 consecutive patients with obesity coming for bariatric surgery at our hospital were enrolled in 2007. Obese subjects had poorer WHOQOL-BREF scores than those of the healthy referents in physical, psychological, and social domains but not in environmental domain $(P<0.05)$. Patients with BMI levels above $32 \mathrm{~kg} / \mathrm{m}^{2}$ had consistently poorer scores in various facets after adjusting for other risk factors.

Conclusions The higher the BMI level the poorer the HRQL. Our findings seem to support the recommendations of Asia-Pacific consensus based on HRQL considerations.

Keywords Morbid obesity - Quality of life .

Bariatric surgery $\cdot$ Roux-en-Y gastric bypass .

WHOQOL-BREF

\section{Introduction}

The prevalence of obesity has increased markedly in the past 20 years, becoming a major public health issue [1-3]. Many obesity-related comorbidities $[4,5]$ have been documented and account for the use of considerable medical resources worldwide. It has been found that the higher one's BMI value, the higher his or her mortality [6, 7]. However, although nonsurgical treatment of obesity such as behavioral and pharmacologic methods have been proved to have an effect on short-term weight loss of approximately 5 to $10 \%$ of body weight [8], these methods are not very effective on long-term resolution of excess body weight and its related comorbidities. Therefore, bariatric surgery plays an increasingly 
important role for this problem $[9,10]$. Bariatric surgery not only helps reduce body weight and decrease comorbidities, but it also causes an improvement in patients' health-related quality of life (HRQOL) [11-14]. However, most, if not all, studies do not control for potential confounding by other risk factors on related quality of life (QOL).

QOL refers not only to health but also to social and environmental status (e.g., social support, income, and education) that can substantially affect well-being. In 1991, the World Health Organization initiated a project to develop a generic QOL instrument in ten countries simultaneously, which led to the World Health Organization Quality of Life (WHOQOL) instrument $[15,16]$. The WHOQOL has two unique features. It encompasses physical, psychological, social, and environment domains comprehensively, and it is a cross-cultural instrument developed for use across different patient groups and in different countries [17]. The WHOQOL group later simplified the standard questionnaire to a short form called the WHOQOL-BREF [18]. It appears to be a sensitive tool to evaluate HRQOL for patients with different diseases [19-22]. Our study aims to compare the HRQOL of patients with morbid obesity coming for bariatric surgery and the healthy general population in Taiwan.

\section{Materials and Methods}

\section{Patients}

This study was approved by the Institutional Ethics Committee of E-Da Hospital, I-Shou University. All patients who came to the Bariatric Center seeking surgical treatment of morbid obesity were invited into this study. The surgery has been regularly performed in this hospital, and we use the laparoscopic Roux-en-Y gastric bypass procedure. We adopted the recommendation of the AsiaPacific consensus [23], which stipulates that to be eligible for bariatric surgery, patients must be between ages 18 and 65 years, and their BMI must fall between 32 and $40 \mathrm{~kg} / \mathrm{m}^{2}$ with obesity-related comorbidities, or their BMI must be more than $40 \mathrm{~kg} / \mathrm{m}^{2}$. Exclusion criteria included previous gastric surgery, a large hiatal hernia, a history of alcohol or substance addiction and poorly controlled non-obesityrelated medical diseases such as unresolved depression. All the patients underwent a preoperation psychiatric interview to determine any mental illnesses or unrealistic expectations for the surgical treatment, and if they had either, they were excluded from this study.

\section{HRQL Questionnaire}

Every subject was asked to complete a validated generic QOL questionnaire in the outpatient clinic of the Bariatric
Center-namely, the WHOQOL-BREF, Taiwan version [24], at 1 month before the operation. The Taiwan version of the WHOQOL-BREF contains four domains (physical, psychological, social, and environmental), including the 26 original items of the WHOQOL-BREF, plus two culturespecific questions as national items of Taiwan. One item addressing "respect from others" was categorized into the social domain and another corresponded to "eating what one likes to eat" and was referred to the environmental domain. The method of application, the scoring procedures, and reference time point (during the last 2 weeks) were the same as for the original WHOQOL-BREF [18]. In brief, each item was scored from 1 to 5 points and a higher score was considered a better QOL. Because the numbers of items are different for each domain, the domain scores were calculated by multiplying the average of the scores of all items in the domain by the same factor of 4 . Therefore, each domain score would have the same range, from 4 to 20 .

\section{Physical Evaluation}

Echocardiography, lung function test, complete blood count, and serum biochemistry profile including AST, ALT, triglycerides, total cholesterol, LDL, HDL, and fasting glucose were performed before the operation. Body weight and height were measured simultaneously. Esophagogastroduodenoscopy was performed to detect peptic ulcer and possible infection with Helicobacter pylori, which would be treated before operation. The presence of diabetes, heart disease, hypertension, asthma, sleep apnea, cancer, and other major comorbidities, as well as education level, marital status, employment, religion, monthly income, and histories of smoking or drinking were recorded.

\section{Reference Population}

A reference group with age- (within 3 years), sex-, municipality-, marriage- and education-matched healthy subjects was randomly sampled from the database of 2001 National Health Interview Survey (NHIS) conducted by the National Health Research Institute and the Bureau of Health Promotion, Department of Health, Taiwan. The 2001 NHIS intended to provide nationwide estimates on health conditions, health behaviors, and usage of medical resources for the Taiwanese population. The WHOQOLBREF, Taiwan version, was one of the tools included in this national survey program. In total, 27,160 eligible persons living in 7,357 households were selected through multistage sampling proportional to household population size in January 2001. It is very unique in the world that this sample could be representative of the national population in age, 
sex, and urbanization index. The final 2001 NHIS data were collected from 25,464 persons living in 6,271 households, with a response rate of $93.8 \%$ by person and $91.4 \%$ by household [25]. A total of 13,083 persons age 20 to 65 years finished the WHOQOL-BREF, Taiwan version. In our study, each morbidly obese patient was matched with two reference subjects with a BMI no greater than $32 \mathrm{~kg} / \mathrm{m}^{2}$ from the national sample, which is the lower limit of an indication for bariatric surgery in the Asia-Pacific consensus [23].

\section{Statistical Analysis}

We first conducted a descriptive analysis and listed and compared the demographic characteristics of the patients and reference subjects. Then, different domains of WHOQOL were summarized and stratified by different ranges of BMI, from less than 25,25 to 32,32 to 35,35 to 40 , and more than 40. A general linear model (GLM) analysis was performed to test the trend of QOL score changes along the above ranges of increased BMI. Multiple linear regression models were constructed by using the summary scores of each domain and individual items as the dependent variables, while the different BMI categories, age, sex, years of education, employment, monthly income, marital status, religion, smoking, alcohol drinking, and comorbidities were included as the independent predictive variables. A forward stepwise strategy was applied to select significant independent variables with $P<0.05$ as the inclusion criterion. All data were collected and analyzed using version 9.0 of SAS software.

\section{Results}

One hundred twenty-one consecutive obese patients came for bariatric surgery at our hospital between January 2007 and November 2007. Seven patients with BMIs less than $32 \mathrm{~kg} / \mathrm{m}^{2}$ were excluded. A total of 224 healthy subjects with BMIs less than $32 \mathrm{~kg} / \mathrm{m}^{2}$ were matched with age (within 3 years), sex, municipality, marriage, and education and were randomly sampled from the database of the 2001 National Health Interview Survey (NHIS) in Taiwan. The demographic and clinical characteristics of 114 patients with morbid obesity and 224 healthy controls are summarized in Table 1. The mean age was 32.0 years, and about two thirds of the patients were not married. Obese subjects were more likely to have personal religions and drinking habits than healthy subjects. All the patients and healthy subjects were classified into five subgroups according to their BMI. While the BMI of all healthy subjects was below $32 \mathrm{~kg} / \mathrm{m}^{2}$, a majority or $64 \%$ of obese subjects had a BMI more than $40 \mathrm{~kg} / \mathrm{m}^{2}$ (Table 2).

Table 1 Demographic characteristics of patients with obesity coming for bariatric surgery, and age-, sex-, municipality-, marriage-, and education-matched healthy control subjects

\begin{tabular}{|c|c|c|c|}
\hline Characteristics & Patients with obesity & Healthy subjects & $P$ value \\
\hline Number of subjects & 114 & 224 & \\
\hline Sex $(\%$ female $)$ & 58.8 & 58.5 & 0.96 \\
\hline Age $($ mean $\pm \mathrm{SD})$ & $32.0 \pm 9.7(18-54)$ & $31.9 \pm 9.5(20-50)$ & 0.94 \\
\hline Weight $(\mathrm{kg} ;$ mean $\pm \mathrm{SD})$ & $123.1 \pm 25.6(80-190)$ & $60.6 \pm 10.8(40-92)$ & $<0.01$ \\
\hline Height $(\mathrm{m} ;$ mean $\pm \mathrm{SD})$ & $162.6 \pm 8.0(150-197)$ & $163.5 \pm 7.0(140-188)$ & $<0.01$ \\
\hline BMI $\left(\mathrm{kg} / \mathrm{m}^{2} ;\right.$ mean $\left.\pm \mathrm{SD}\right)$ & $43.1 \pm 7.9(32-67)$ & $22.6 \pm 3.3(17-31)$ & $<0.01$ \\
\hline Percent married & 34.2 & 34.4 & 0.98 \\
\hline \multicolumn{4}{|l|}{ Education (years) } \\
\hline$<6$ & $2.6 \%$ & $3.1 \%$ & \\
\hline $6-12$ & $33.3 \%$ & $33.9 \%$ & \\
\hline$>12$ & $64.1 \%$ & $63.0 \%$ & \\
\hline Percent employment & 79.8 & 70.1 & 0.06 \\
\hline Percent religion & 71.9 & 59.8 & 0.03 \\
\hline Percent smoking & 28.9 & 28.6 & 0.94 \\
\hline Percent drinking & 42.1 & 24.1 & $<0.01$ \\
\hline Monthly income & & & 0.09 \\
\hline None & $18.6 \%$ & $23.2 \%$ & \\
\hline NT $<20,000$ & $27.2 \%$ & $18.8 \%$ & \\
\hline NT $20,000-\mathrm{NT} 40,000$ & $29.8 \%$ & $34.2 \%$ & \\
\hline NT 40,000-NT60,000 & $7.9 \%$ & $14.7 \%$ & \\
\hline $\mathrm{NT}>60,0000$ & $18.4 \%$ & $9.3 \%$ & \\
\hline$\%$ with comorbidities & 54.3 & 0 & $<0.01$ \\
\hline
\end{tabular}


Table 2 Comparison of QOL from four domains, overall score ratings between patients with obesity coming for bariatric surgery, and age-, sex-, municipality-, marriage-, and education-matched healthy control subjects based on the WHOQOL-BREF (mean $\pm \mathrm{SD}$ )

\begin{tabular}{|c|c|c|c|c|c|c|}
\hline \multirow[t]{2}{*}{ Domains } & \multicolumn{2}{|c|}{ Healthy subjects } & \multicolumn{3}{|c|}{ Patients coming for bariatric surgery } & \multirow[t]{2}{*}{$P$ value } \\
\hline & $\begin{array}{l}\mathrm{BMI}<25 \\
(n=165)\end{array}$ & $\begin{array}{l}\mathrm{BMI} \leq 25 \text { to }<32 \\
(n=59)\end{array}$ & $\begin{array}{l}\mathrm{BMI} \leq 32 \text { to }<35 \\
(n=19)\end{array}$ & $\begin{array}{l}\mathrm{BMI} \leq 35 \text { to }<40 \\
(n=22)\end{array}$ & $\begin{array}{l}\mathrm{BMI} \geq 40 \\
(n=73)\end{array}$ & \\
\hline Q1 overall QOL* & $3.45 \pm 0.65$ & $3.36 \pm 0.58$ & $3.32 \pm 0.75$ & $3.18 \pm 0.73$ & $2.96 \pm 0.82$ & $<0.01$ \\
\hline Q2 overall health* & $3.55 \pm 0.63$ & $3.58 \pm 0.59$ & $2.74 \pm 0.93$ & $2.41 \pm 0.85$ & $2.25 \pm 0.80$ & $<0.01$ \\
\hline Physical* & $15.65 \pm 1.72$ & $15.22 \pm 2.27$ & $14.53 \pm 2.10$ & $14.42 \pm 2.23$ & $13.49 \pm 2.25$ & $<0.01$ \\
\hline Psychological* & $14.07 \pm 2.25$ & $13.62 \pm 2.25$ & $12.18 \pm 3.74$ & $11.48 \pm 2.23$ & $11.68 \pm 2.69$ & $<0.01$ \\
\hline Social* & $14.54 \pm 2.11$ & $14.18 \pm 2.04$ & $13.58 \pm 2.73$ & $12.72 \pm 2.88$ & $13.47 \pm 2.67$ & 0.01 \\
\hline Environmental & $13.68 \pm 1.98$ & $13.17 \pm 2.15$ & $13.99 \pm 2.41$ & $14.46 \pm 1.53$ & $13.46 \pm 2.19$ & 0.10 \\
\hline
\end{tabular}

BMI values in $\mathrm{kg} / \mathrm{m}^{2}$

$* P<0.05$, significant for trend

\section{GLM Analysis for the Trend of WHOQOL-BREF Score Changes}

The GLM model showed a significant trend of decreasing scores in overall health, overall QOL, physical, psychological, and social domains along with increasing BMI among healthy subjects and patients. The mean scores of the WHOQOL-BREF for the obese group were similar to those of the healthy subjects in the environmental domain.

Multiple Linear Regression Analysis of HRQL Scores in Patients with Morbid Obesity and Healthy Subjects

To improve statistical efficiency, the educational status was classified as high education ( $>12$ years) and low education $(\leq 12$ years). Low economic status was defined as the monthly income less than 20,000 NT dollars (570 US\$). Results of multiple regression analysis for different domain scores of WHOQOL-BREF showed that patients with morbid obesity had lower scores in the physical, psychological, and social domains (Table 3). However, QOL scores in the environmental domain were the same as those of the general population. Employment and education are the major factors associated with increased HRQL scores.
High education and high monthly income were also associated with increased scores in the environmental domain.

Analyses of HRQL Scores in Facets of Each Domain by Multiple Linear Regression Modeling

Table 4 summarizes results of multiple linear regression analysis for HRQL scores in individual facets of each domain. In the physical domain, obese subjects had significantly poorer scores in pain and discomfort, energy and fatigue, sleep and rest, mobility, activities of daily living, and dependence on medication or treatments after adjustment of other risk factors. They also showed lower scores in negative feelings, body image and appearance, self-esteem, and difficulty in thinking, learning, memory, and concentration in the psychological domain, as well as sexual life and being respected and accepted in the social domain. However, they did not appear to have lower scores in most facets of the environmental domain.

After adjusting for other risk factors, we found that employment significantly increased HRQL scores in facets of activities of daily living, thinking, learning, memory and concentration, self-esteem, negative feelings, social support,

Table 3 Regression coefficients and standard error (in parentheses) based on multiple linear regression analysis of HRQL and determinants in patients with obesity coming for bariatric surgery, and age-, sex-, municipality-, marriage-, and education-matched healthy control subjects

\begin{tabular}{|c|c|c|c|c|}
\hline & Physical & Psychological & Social & Environmental \\
\hline Constant & $14.42 * *(0.24)$ & $12.71 * *(0.32)$ & $13.88 * *(0.25)$ & $12.67 * *(0.21)$ \\
\hline Obesity for bariatric surgery (yes/no) & $-1.78 * *(0.22)$ & $-2.34 * *(0.28)$ & $-1.00 * *(0.26)$ & - \\
\hline Employment (yes/no) & $0.87 * *(0.24)$ & $1.09^{* *}(0.30)$ & $1.06^{* *}(0.28)$ & - \\
\hline Education $(>12$ years $/ \leq 12$ years $)$ & $0.79 * *(0.22)$ & $0.76^{*}(0.28)$ & - & $0.88 *(0.23)$ \\
\hline Monthly income $(\geq$ NT $20,000 /<$ NT 20,000$)$ & - & - & - & $0.65^{* *}(0.22)$ \\
\hline Smoke (yes/no) & - & - & $-0.64 *(0.28)$ & - \\
\hline
\end{tabular}

$* P<0.05, * * P<0.005$ 


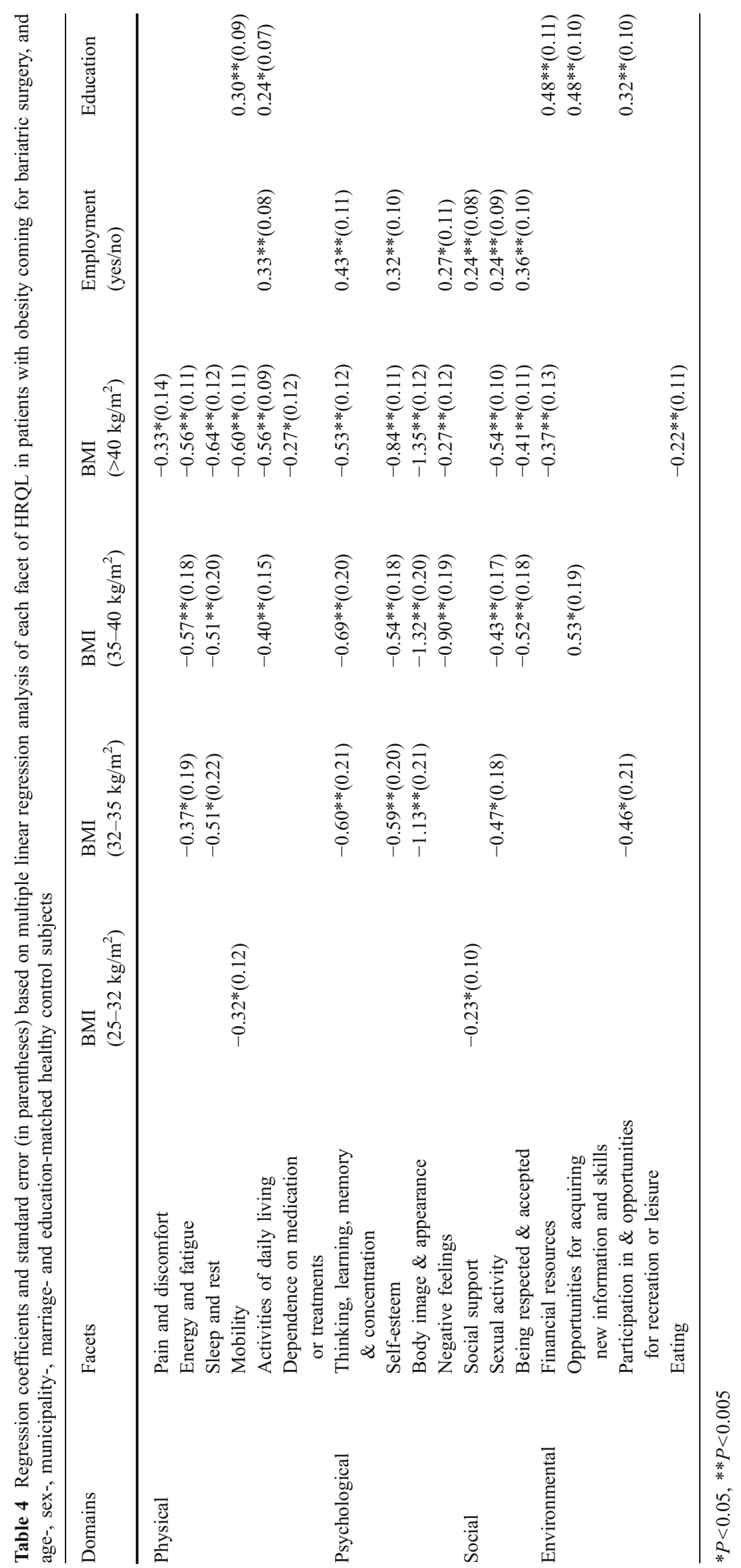


sexual activity, and being respected and accepted. Similarly, high education increased the HRQL scores in facets of mobility and activities of daily living, financial resources, opportunities for acquiring new information and skills, and participation in and opportunities for recreation. A higher monthly income increased the HRQL scores in mobility, working capacity, thinking, learning, memory and concentration, personal relationships, home environment, financial resources, and opportunities for acquiring new information and skills. In the social domain, smoking was associated with lower scores in social support and sexual activity.

\section{Discussion}

Although there have been reports of impaired QOL among patients seeking bariatric surgery, none have seemed to have a comprehensive control for potential confounding by other risk factors $[14,26,27]$. Our study is the first to have included healthy control subjects and adjusted for risk factors affecting QOL scores including age, sex, education, municipality, and marriage in our multiple regression analysis. After controlling for other risk factors, we have demonstrated that morbidly obese subjects suffer from poor QOL in physical, psychological, and social domains and various facets as shown in Tables 2 and 4; there is a general tendency that the bigger the BMI, the poorer the scores of affected domains and facets.

In the West, the indication for bariatric surgery is a BMI between 35 and $40 \mathrm{~kg} / \mathrm{m}^{2}$ with comorbidity, or $\mathrm{BMI} \geq$ $40 \mathrm{~kg} / \mathrm{m}^{2}$ by NIH criteria [28, 29]. However, for ethnic considerations, the diagnostic criteria of obesity in Asia has been modified by WHO, with two classes of obesity: obese class $\mathrm{I}=\mathrm{BMI}>25 \mathrm{~kg} / \mathrm{m}^{2}$, and obese class $\mathrm{II}=\mathrm{BMI}>30 \mathrm{~kg} / \mathrm{m}^{2}$ (equal to the subgroup of BMI $>35 \mathrm{~kg} / \mathrm{m}^{2}$ in the West). Thus, there has been a debate about what cutoff level of BMI is the most appropriate indication for bariatric surgery $[23,28]$. In our study of subjects from Asian countries, Tables 2 and 4 consistently indicate that a BMI $>32$ began to show significantly decreased scores for physical, psychological, and social domains and related facets after adjustment for other risk factors. Our findings seem to support the recommendation of the consensus meeting from Asian-Pacific scholars [23] based on QOL considerations. Considering the additional benefit of controlling type 2 diabetes and other comorbidities by this type of surgery, we recommend that future clinical trials include patients with BMIs lower than $32 \mathrm{~kg} / \mathrm{m}^{2}$.

Previous studies on QOL of people with morbid obesity have reported the negative impacts on physical and psychological domains based on the SF-36 [14, 26, 27, 30], which is a generic instrument. The condition-specific instrument of bariatric analysis and reporting outcome system (BAROS) [31] further demonstrated that obese subjects reported less pleasant sexual activity. We administered the Taiwan version of WHOQOL-BREF in this study, which not only corroborates the above findings but also indicates that such patients were less likely accepted or respected by others in the society and suffered from poor QOL in the social domain (Tables 3 and 4). In Asian or ethnic Chinese culture, being respected is one of the major concerns in people's life and not being respected would decrease their QOL [24].

Taiwan established the National Health Insurance (NHI) system in 1995, which includes more than $96 \%$ of Taiwan's population and covers emergency, outpatient and inpatient care, laboratory tests, diagnostic imaging and medication; citizens pay an average of 44 US dollars in insurance premiums every month [32]. However, the cost of bariatric surgery is about 3,750 US dollars for one patient and is still not covered by the NHI. Future studies are needed to evaluate the cost-effectiveness of this surgery, or the number of dollars per quality-adjusted life year or per life year, in comparison with other treatments so that recommending inclusion of bariatric surgery to the regular reimbursement schedule can be justified.

In conclusion, the HRQL for patients with morbid obesity in Taiwan is worse than for those of the general population, except in the environmental domain, after controlling for other risk factors, including age, sex, marriage, employment, and education. The higher the BMI level is above 32, the poorer are the domain and facet scores. Given the potential benefit of preventing comorbidities, including type 2 diabetes mellitus, we propose that such a surgical procedure might be considered in the future for patients with morbid obesity and those with a BMI less than 32 .

Disclosure None of the authors holds any significant financial interest in the product discussed that would represent a conflict of interest.

Open Access This article is distributed under the terms of the Creative Commons Attribution Noncommercial License which permits any noncommercial use, distribution, and reproduction in any medium, provided the original author(s) and source are credited.

\section{References}

1. Prentice AM. The emerging epidemic of obesity in developing countries. Int J Epidemiol. 2006;35:93-9.

2. Ogden CL, Carroll MD, Curtin LR, et al. Prevalence of overweight and obesity in the United States, 1999-2004. JAMA. 2006;295:1549-55.

3. Sturm R. Increases in clinically severe obesity in the United States, 1986-2000. Arch Intern Med. 2003;163:2146-8.

4. Bray GA. Medical consequences of obesity. J Clin Endocrinol Metab. 2004;89:2583-9. 
5. Li Z, Bowerman S, Heber D. Health ramifications of the obesity epidemic. Surg Clin North Am. 2005;85:681-701.

6. Adams KF, Schatzkin A, Harris TB, et al. Overweight, obesity, and mortality in a large prospective cohort of persons 50 to 71 years old. N Engl J Med. 2006;355:763-8.

7. Flegal KM, Graubard BI, Williamson DF, et al. Excess deaths associated with underweight, overweight, and obesity. JAMA. 2005;293:1861-7.

8. Delinsky SS, Latner JD, Wilson GT. Binge eating and weight loss in a self-help behavior modification program. Obesity (Silver Spring). 2006;14:1244-9.

9. Maggard MA, Shugarman LR, Suttorp M, et al. Meta-analysis: surgical treatment of obesity. Ann Intern Med. 2005;142:547-59.

10. Davis MM, Slish K, Chao C, et al. National trends in bariatric surgery, 1996-2002. Arch Surg. 2006;141:71-4; discussion 75.

11. Engel SG, Crosby RD, Kolotkin RL, et al. Impact of weight loss and regain on quality of life: mirror image or differential effect? Obes Res. 2003;11:1207-13.

12. Glinski J, Wetzler S, Goodman E. The psychology of gastric bypass surgery. Obes Surg. 2001;11:581-8.

13. Christou NV, Sampalis JS, Liberman M, et al. Surgery decreases long-term mortality, morbidity, and health care use in morbidly obese patients. Ann Surg. 2004;240:416-23; discussion 423-4.

14. Livingston EH, Fink AS. Quality of life: cost and future of bariatric surgery. Arch Surg. 2003;138:383-8.

15. The World Health Organization Quality of Life Assessment (WHOQOL): development and general psychometric properties. Soc Sci Med. 1998;46:1569-85.

16. Study protocol for the World Health Organization project to develop a Quality of Life assessment instrument (WHOQOL). Qual Life Res. 1993;2:153-9.

17. Anderson RT, Aaronson NK, Bullinger M, et al. A review of the progress towards developing health-related quality-of-life instruments for international clinical studies and outcomes research. Pharmacoeconomics. 1996;10:336-55.

18. Development of the World Health Organization WHOQOL-BREF quality of life assessment. The WHOQOL Group. Psychol Med. $1998 ; 28: 551-8$.
19. Yang SC, Kuo PW, Wang JD, et al. Quality of life and its determinants of hemodialysis patients in Taiwan measured with WHOQOL-BREF(TW). Am J Kidney Dis. 2005;46:635-41.

20. Hsiung PC, Fang CT, Chang YY, et al. Comparison of WHOQOL-BREF and SF-36 in patients with HIV infection. Qual Life Res. 2005;14:141-50.

21. Hsu C, Wang JD, Hwang JS, et al. Survival-weighted health profile for long-term survivors of acute myelogenous leukemia. Qual Life Res. 2003;12:503-17.

22. Liou HH, Chen RC, Chen CC, et al. Health related quality of life in adult patients with epilepsy compared with a general reference population in Taiwan. Epilepsy Res. 2005;64:151-9.

23. Lee WJ, Wang W. Bariatric surgery: Asia-Pacific perspective. Obes Surg. 2005;15:751-7.

24. Yao G, Chung CW, Yu CF, et al. Development and verification of validity and reliability of the WHOQOL-BREF Taiwan version. J Formos Med Assoc. 2002;101:342-51.

25. Lin SH. Field collection and completeness of data in the National Health Interview Survey: NHIS Brief Communication No. 4, 2002.

26. Choban PS, Onyejekwe J, Burge JC, et al. A health status assessment of the impact of weight loss following Roux-en-Y gastric bypass for clinically severe obesity. J Am Coll Surg. 1999;188:491-7.

27. Nguyen NT, Goldman C, Rosenquist CJ, et al. Laparoscopic versus open gastric bypass: a randomized study of outcomes, quality of life, and costs. Ann Surg. 2001;234:279-89; discussion 289-91.

28. NIH conference. Gastrointestinal surgery for severe obesity. Consensus Development Conference Panel. Ann Intern Med. 1991;115:956-61.

29. Flegal KM, Carroll MD, Ogden CL, et al. Prevalence and trends in obesity among US adults, 1999-2000. JAMA. 2002;288:1723-7.

30. Dymek MP, Le Grange D, Neven K, et al. Quality of life after gastric bypass surgery: a cross-sectional study. Obes Res. 2002;10:1135-42.

31. Oria HE, Moorehead MK. Bariatric analysis and reporting outcome system (BAROS). Obes Surg. 1998;8:487-99.

32. Cheng TM. Taiwan's new national health insurance program: genesis and experience so far. Health Aff (Millwood). 2003;22:61-76. 